\title{
Corrigendum: A solid-solid phase transition in carbon dioxide at high pressures and intermediate temperatures
}

Jinjin Li, Olaseni Sode, Gregory A. Voth \& So Hirata

Nature Communications 4:2647 doi: 10.1038/ncomms3647 (2013); Published 22 Oct 2013; Updated 6 Nov 2015

In Table 1 of this article, the values of lattice constants $a$ and $b$ of $\mathrm{CO}_{2}$-III calculated by second-order Møller-Plesset perturbation theory ('4.33' and ' 4.63 ' in the rightmost column) were inadvertently swapped. The correct version of Table 1 appears below.

\begin{tabular}{|c|c|c|c|}
\hline Parameter $^{\star}$ & Expt. $^{\dagger}$ & Calc. ${ }^{\ddagger}$ & Calc. ${ }^{\S}$ \\
\hline$a(1) / \AA$ & $4.939 \pm 0.010$ & 4.92 & 4.91 \\
\hline$a(I I I) / \AA$ & $4.330 \pm 0.015$ & 4.17 & 4.63 \\
\hline$b(I I I) / \AA$ & $4.657 \pm 0.005$ & 4.65 & 4.33 \\
\hline$c(I I I) / \AA$ & $5.963 \pm 0.009$ & 6.02 & 5.80 \\
\hline$\varphi(I I I) /$ degree & 52 & 51.9 & 54.8 \\
\hline
\end{tabular}

